\title{
Multidisciplinary Design Optimization of Wing Shape for a Small Jet Aircraft Using Kriging Model
}

\author{
Takayasu Kumano ${ }^{*}$, Shinkyu Jeong ${ }^{\dagger}$, Shigeru Obayashi ${ }^{\ddagger}$ \\ Institute of Fluid Science, Tohoku University, Sendai 980-8577, Japan, \\ Yasushi Ito ${ }^{\S}$ \\ University of Alabama at Birmingham, Birmingham 35294-4461, USA, \\ Keita Hatanaka", and Hiroyuki Morino \# \\ Mitsubishi Heavy Industries, Ltd., Nagoya 455-8515, Japan
}

\begin{abstract}
In this paper, a practical Multidisciplinary Design Optimization (MDO) system for an aircraft design is developed. The MDO system is based on the integration of computational fluid dynamics (CFD) codes and the NASTRAN based aeroelastic-structural interface code. Kriging model is employed to save the computational time of objective function evaluation in Multi-Objective Genetic Algorithm (MOGA). As a result of optimization, several nondominated solutions, indicating the trade-off among the drag, the structural weight, the drag divergence and the pitching moment, have been found.
\end{abstract}

\section{Introduction}

A conventional aircraft design has been emphasized mainly on an aerodynamic performance and auxiliary design revisions have been done to satisfy other design requirements from the structure and the system. However, based on such aerodynamically oriented design, it is difficult to design the aircraft considering interdisciplinary trade-off, which is particularly remarkable between aerodynamic (minimum drag) and structural (minimum weight) performance. Recently, researches of MDO for a realistic aircraft design are conducted by industries and universities ${ }^{1,2}$.

In Japan, a 5-year R\&D project has been in progress toward the development of an environmentally friendly high performance regional jet aircraft under auspice from New Energy Development Organization of Japan (NEDO) since 2003. In the project, new technical features have been investigated by industry-government-university cooperation $^{3}$. The conceptual image of a regional jet aircraft is shown in Fig. 1. For the success of the project, a practical MDO system for regional jet wing design is required.

Chiba et al. developed the proto-type MDO system using MOGA and high-fidelity aero-structure analysis models, such as unstructured Euler/Navier-Stokes CFD solver and NASTRAN ${ }^{4,5}$. Though this MDO system was successfully applied to the regional jet wing design considering aerodynamic, aeroelastic and structural design, the system revealed following problems:

i) Inadequate airfoil shape definition

ii) Tremendous computational time for function evaluation

\footnotetext{
${ }^{*}$ Graduate Student, Institute of Fluid Science, Member AIAA.

${ }^{\dagger}$ Research Associate, Institute of Fluid Science, Member AIAA.

* Professor, Institute of Fluid Science, Associate Fellow AIAA.

$\S$ Postdoctoral Fellow, Department of Mechanical Engineering, AIAA Member.

" Research Engineer, Nagoya Aerospace Systems.

\# Research Engineer, Nagoya Aerospace Systems.
} 
iii) Deficiency in CFD mesh generation

In this study, the proto-type MDO system is improved to overcome these problems.

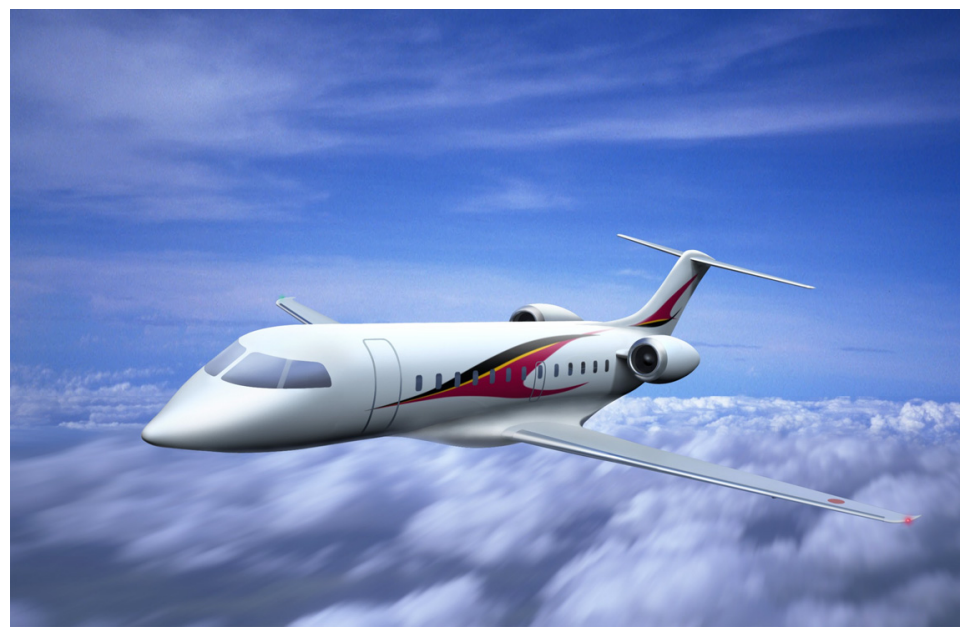

Figure 1. Environmentally friendly high performance regional jet aircraft.

\section{Improvements of MDO System}

\section{A. Improvement of Airfoil Shape Definition Technique}

In the proto-type MDO system, the modified PARSEC method ${ }^{6}$ (Fig. 2) is used because the number of variables is very small and the variables are directly associated with aerodynamic performance. However, this method often fails to express a detailed shape of airfoil, especially near the leading-edge region. In this study, NURBS ${ }^{7,8}$ (Fig. 3 ) technique employing the B-spline function is adopted here. A total number of the design variables is 26. Figure 4 shows the result of the airfoil expression by PARSEC and NURBS. Figure 5 compares the root mean square (RMS) of the difference between original airfoil and airfoil defined by PARSEC and NURBS. According to Fig. 5, NURBS expresses the original airfoil more precisely than PARSEC.

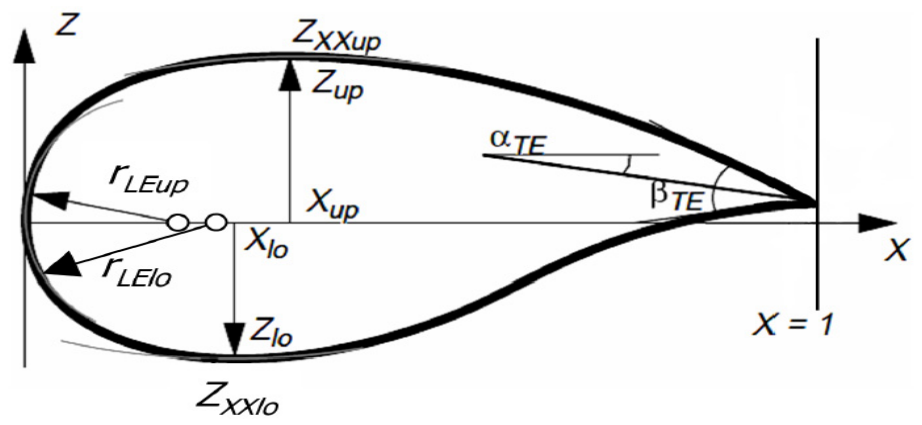

Figure 2. Airfoil defined by PARSEC. 


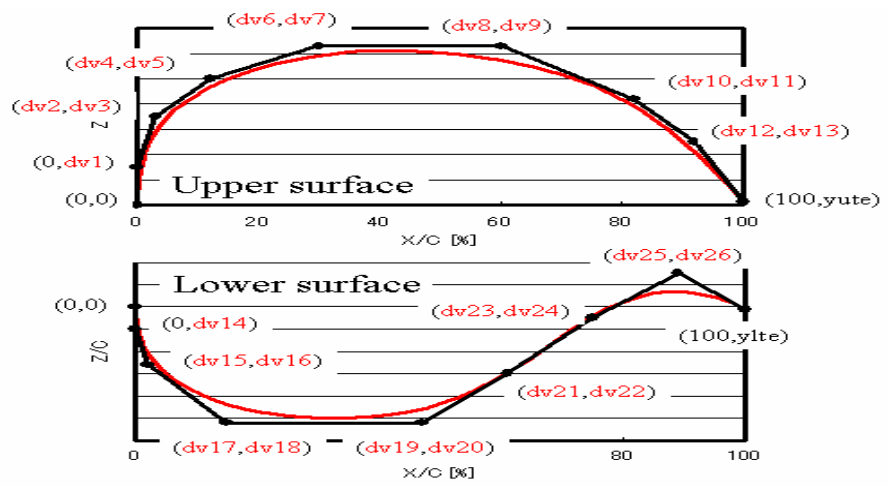

Figure 3. Airfoil defined by NURBS.

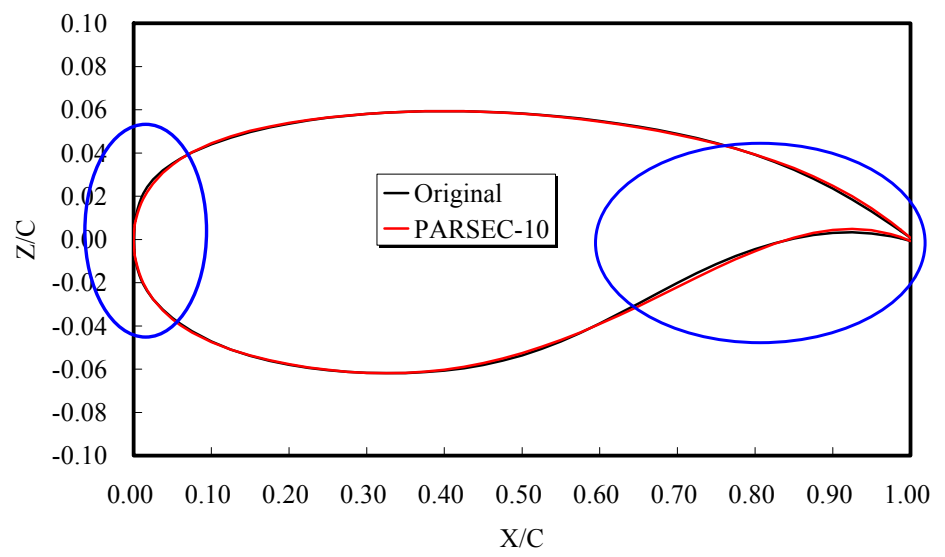

(a)PARSEC

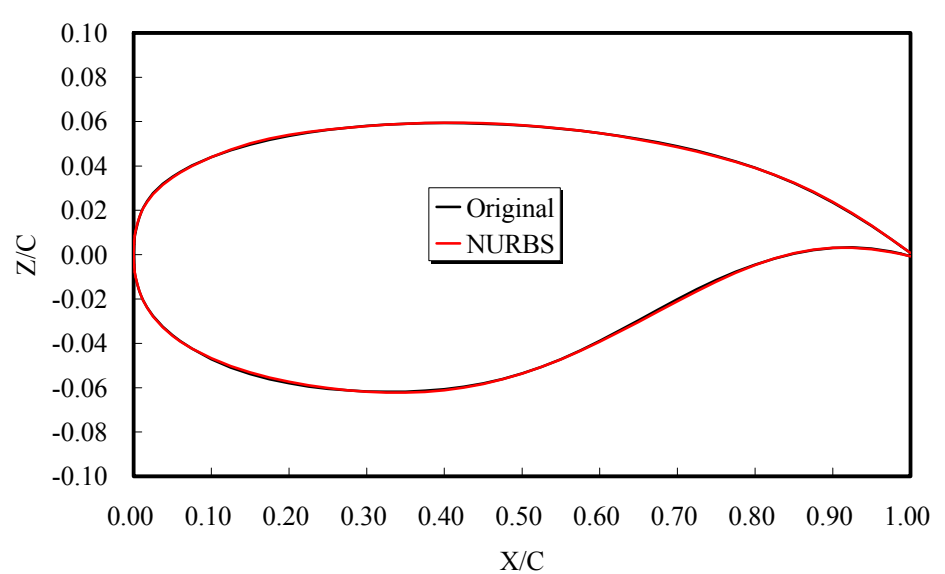

(b) NURBS

Figure 4. Result of airfoil shape expression. 


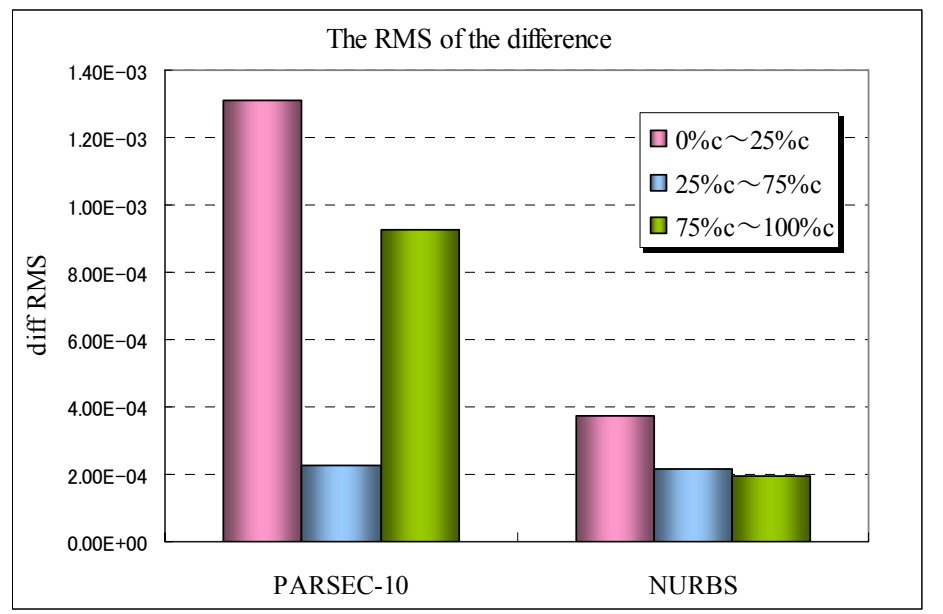

Figure 5. RMS of geometry difference between original airfoil and the airfoil defined by PARSEC and NURBS.

\section{B. Kriging Model}

In the proto-type system, time-consuming high fidelity solvers were used for objective function evaluations in Multi-Objective Genetic Algorithm (MOGA). Even with a high performance supercomputer facility, it required a tremendous computational time.

To make the system practical, an approximation model is introduced in the optimization. The approximation model used in this study is the Kriging model ${ }^{9,}{ }^{10}$. This model, developed in the field of spatial statistics and geostatistics, predicts the distribution of function values at unknown points instead of the function values themselves. From the distribution of function values, both function values and their uncertainty at unknown points can be estimated as shown in Fig. 6. By using these values, the balanced local and global search is possible. This concept is expressed as the criterion 'Expected Improvement (EI) ${ }^{11}$. EI indicates the probability being superior to the current optimum in the design space. By selecting the maximum EI point as an additional sample point of the Kriging model, the improvement of model accuracy and the exploration of the optimum can be achieved at the same time.

In this research, drastic reduction of computational cost has been realized by replacing the time-consuming high fidelity solvers with the Kriging approximation models. The overall optimization procedure using Kriging model is shown in Fig. 7:

(a) Initial sample points distributed uniformly in the design space are selected by the Latin Hypercube Sampling ${ }^{12}$.

(b) Modified full potential calculation (this full potential calculation is corrected based on the database constructed with the Navier-Stokes calculation) is performed, and the initial Kriging models are constructed for all objective functions.

(c) MOGA operations are performed on Kriging models as

- Generation of initial population

- Evaluation of expected improvement (EI)

- Selection parents

- Crossover and mutation

- Evaluation of new individuals

- When the generation exceeds 100, additional sample points (Nadd) are selected from the non-dominated solutions of EIs 
(d) Kriging model is reconstructed as

- Evaluation of the additional sample points with modified full-potential CFD solver and Euler CFD solver

- Correction in the objective function values if necessary

- Construction of new Kriging models with $\mathrm{N}=\mathrm{N}+$ Nadd sample points based on corrected objective function values

(e) Go back to (c) process

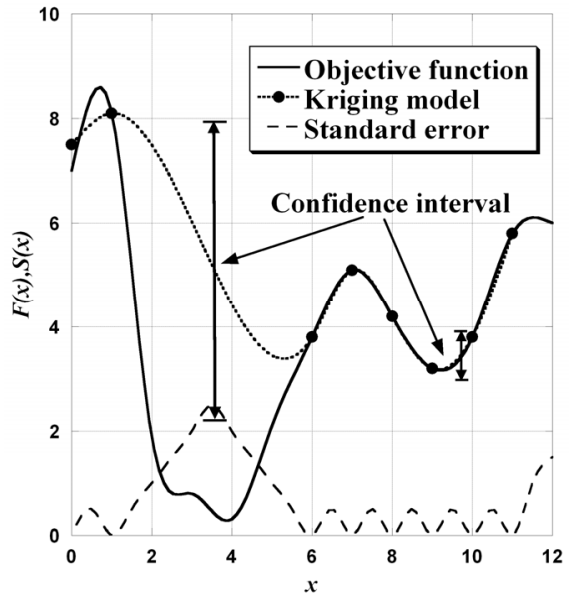

Figure 6. Predicted value and the standard error of the Kriging model.

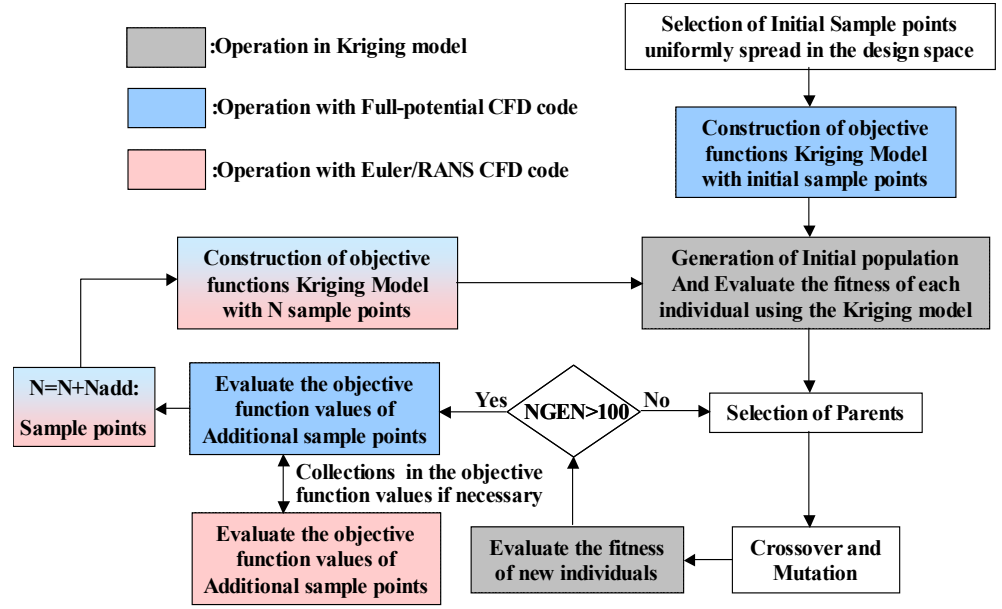

Figure 7. Optimization using the Kriging model.

\section{Automatic Computational Mesh Generation}

In the previous system, surface spline function of geometry deviation $(\Delta \mathrm{z})$ was used for the modification of the wing shape (surface mesh), and then the volume mesh was modified using the unstructured dynamic mesh method ${ }^{13}$, ${ }^{14}$. However, this method sometime produced the distorted surface mesh around the leading edge as shown in Fig. 8. In this case, the system discards the geometry and generates another geometry using MOGA until a valid CFD mesh 
is obtained. Consequently, this process may lead the optimization to an undesirable direction. Therefore, in this study, a speedy and robust surface mesh generation system have been developed. Wing-body configuration surface mesh is automatically generated in the following steps.

(a) Generate the wing geometry that satisfies the design constrains.

(b) Extract the intersection line between the wing and the body.

(c) Delete the wing geometry which is inside the body and unite the wing and the body.

(d) Input mesh point distributions along created ridges.

(e) Generate unstructured surface mesh. The volume mesh is automatically generated by TAS-Mesh ${ }^{15,16}$.

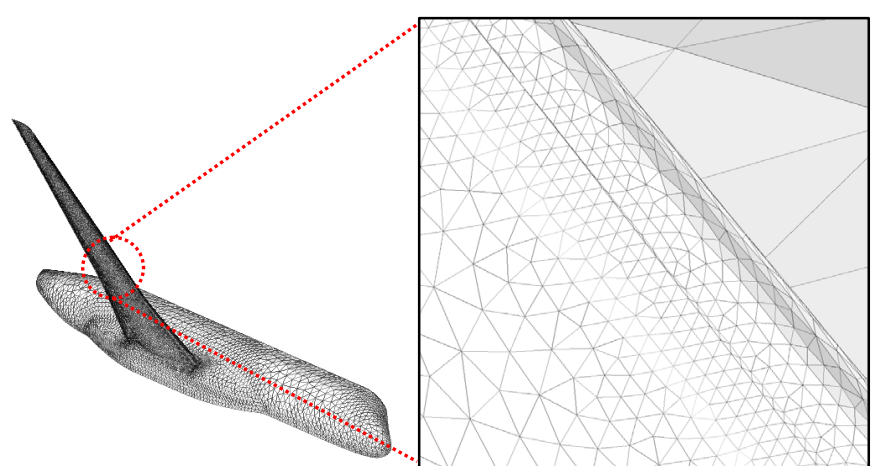

Figure 8. Example of distorted mesh around the leading-edge.

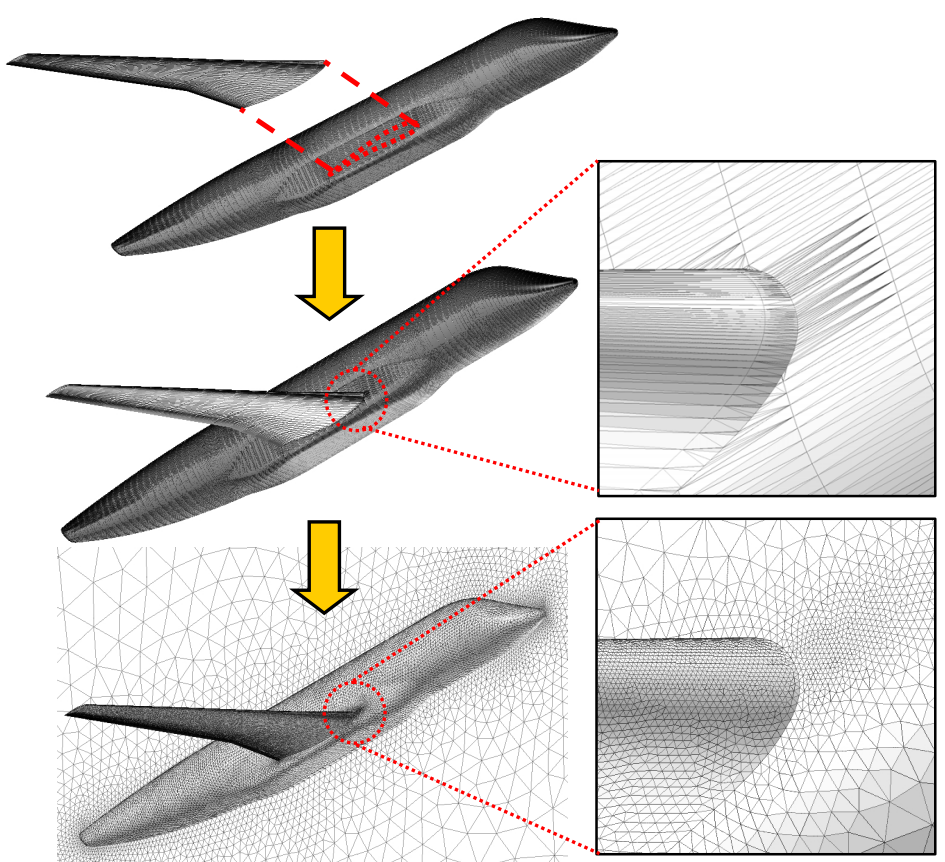

Figure 9. Automatic wing-body mesh generation system.

6

American Institute of Aeronautics and Astronautics 


\section{Revised MDO System}

Flowchart of the revised MDO system is shown in Fig. 10. This MDO system consists of the mesh generation module, CFD \& CSD module, and Kriging model \& Optimizer module.

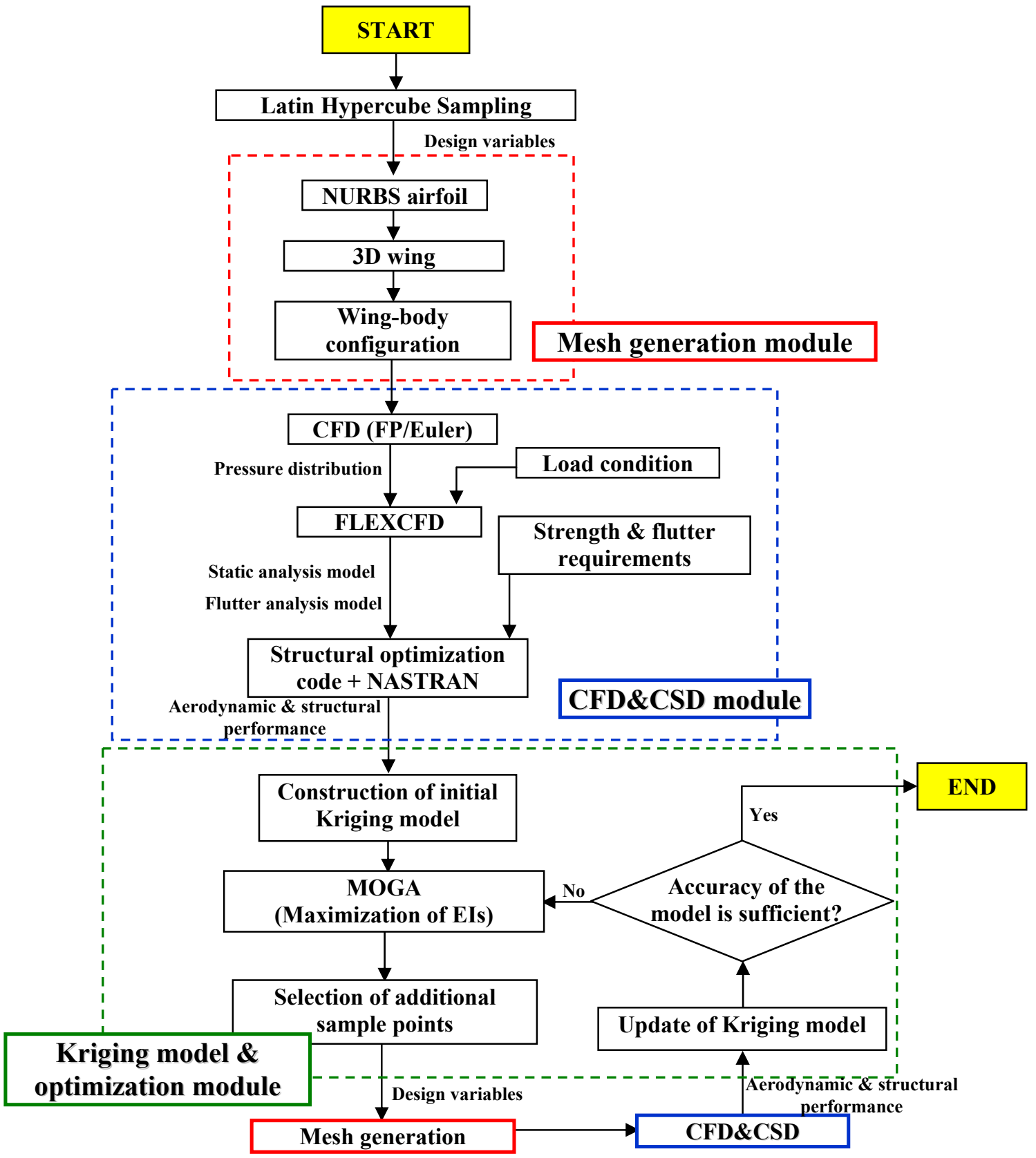

Figure 10. Flowchart of the revised MDO system. 
In order to evaluate aerodynamic and structural performance, CFD\&CSD module in Fig. 10 is performed. The procedure is as followings:

(a) Full potential analysis is performed for all Kriging sample points and Euler analysis is performed for several points to validate the full potential analysis.

(b) Using the pressure distribution obtained from FP/Euler analysis, structural and flutter analysis models are generated by FLEXCFD which is an aeroelastic-structural interface code.

(c) Structural optimization to minimize wing weight that satisfies the strength and flutter requirement is conducted.

Figure 11 shows the CFD and CSD meshes. In the CFD-CSD coupling module, structural optimization of a wing box is performed to realize minimum weight with constraints of strength and flutter requirements. Given the wing outer mold line for each individual, the finite element model of wing box is generated automatically by the FEM generator for the structural optimization. The wing box model mainly consists of shell elements representing skin, spar and rib, and other wing components are modeled using concentrated mass element. In the present study, MSC. NASTRAN ${ }^{4}$ which is a high-fidelity commercial software is employed for the structural and aeroelastic evaluations.

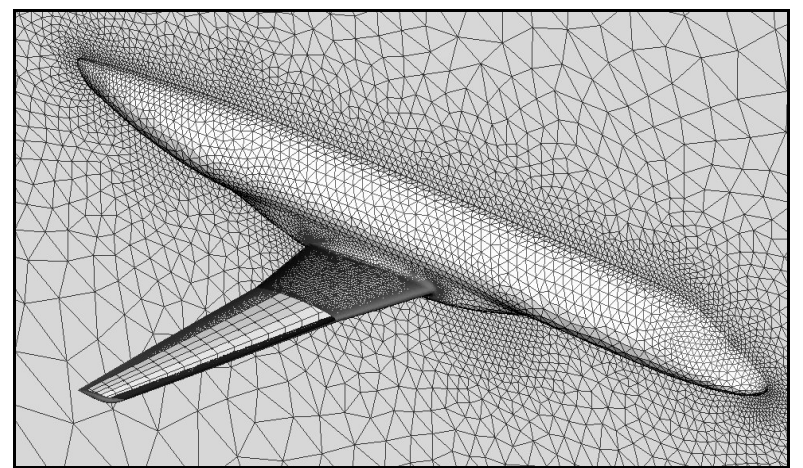

Figure 11. CFD and CSD meshes.

\section{Optimization Problem}

Using the revised MDO design system, the aero-structural wing shape design is performed here.

\section{A. Definition of Optimization Problem}

$<$ Objective functions $>$

Minimize

- Drag at the cruising condition

- Drag divergence between cruising and off-design condition

- Pitching moment at the cruising condition

- Structural weight of the main wing

\section{$<$ Design variables $>$}

- Airfoil shapes at 4 spanwise sections $(\eta=0.1,0.35,0.7$ and 1.0$) \rightarrow 26$ variables $($ NURBS $) \times 4$ sections $=$ 104 variables

- Twist angles at 5 sections $=5$ variables

$\underline{109 \text { variables in total }}$ 
$<$ Constraints $>$

- Rear spar heights $>$ Required values

- Strength and flutter margin $>$ Required values

\section{B. Optimization Results}

In this optimization, the update of Kriging model has been performed in five times. In total 160 sample points were used. Figure 12 shows performances of the baseline configuration and those of additional sample points after several iteration steps. As iteration progresses, individuals move toward the optimum direction. It means that the additional sample points for update are correctly selected. One of the non-dominated solutions (Point A in Fig. 12) is found to be reduced by 6.2 counts in drag, 0.4 counts in drag divergence, 79.4 counts in pitching moment, and $74.0 \mathrm{~kg}$ in wing weight compared with the baseline shape.

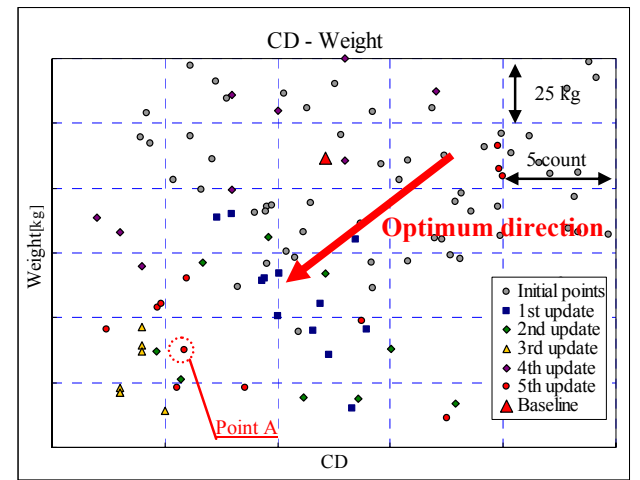

(a) Drag - Wing weight

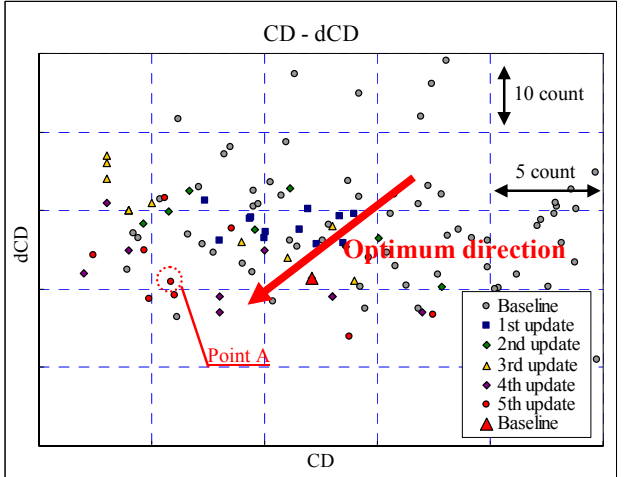

(c) Drag - Drag divergence

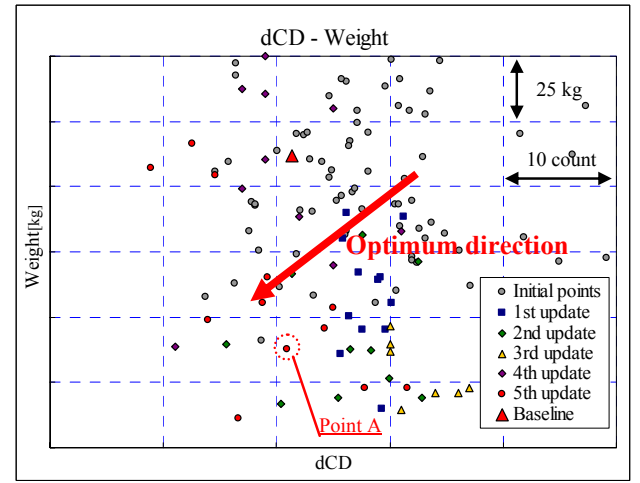

(e) Drag divergence - Wing weight

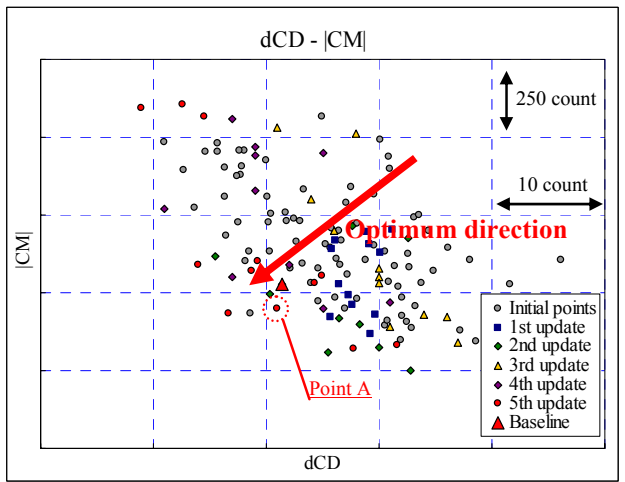

(b) Drag divergence - Pitching moment

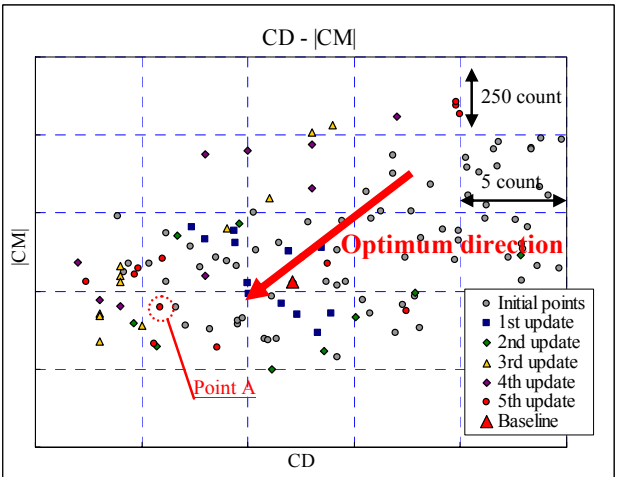

(d) Drag - Pitching moment

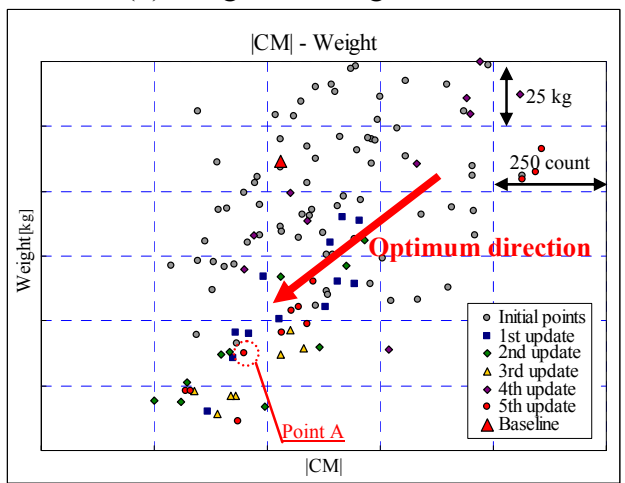

(f) Pitching moment - Wing weight

Figure 12. Optimization results (full potential analysis).

9

American Institute of Aeronautics and Astronautics 
If the optimization problem has less than three objectives, trade-offs relation can be visualized by using conventional two or three-dimensional plots. However, if there are more than three objectives, an advanced visualization technique which can show the trade-off relation of high-dimensional data is needed. In the present study, Self-Organizing Map (SOM) proposed by Kohonen ${ }^{17}$ was employed to identify the trade-off relations between objectives. This method has been applied to data mining of aerodynamic design space ${ }^{18}$.

All of the non-dominated solutions have been projected onto the two-dimensional map of SOM. Figure 13 shows the resulting SOM with 15 clusters considering the four objectives. Furthermore, Fig. 14 shows the same SOM colored by the four objectives, respectively. These color figures show that the SOM indicated in Fig. 13 can be grouped as follows:

The lower right corner corresponds to the designs with high drag and low drag divergence. The lower left corner corresponds to those with high drag divergence and low wing weight. Figure 14(b) and Fig. 14(c) show that there is a trade-off between drag divergence and pitching moment. Furthermore, Figure 14(b) and Fig. 14(d) show that there is a trade-off between drag divergence and wing weight. As the coloring in Fig. 14(a) is similar to that in Fig. 14(c), there was not a severe trade-off between drag and pitching moment.

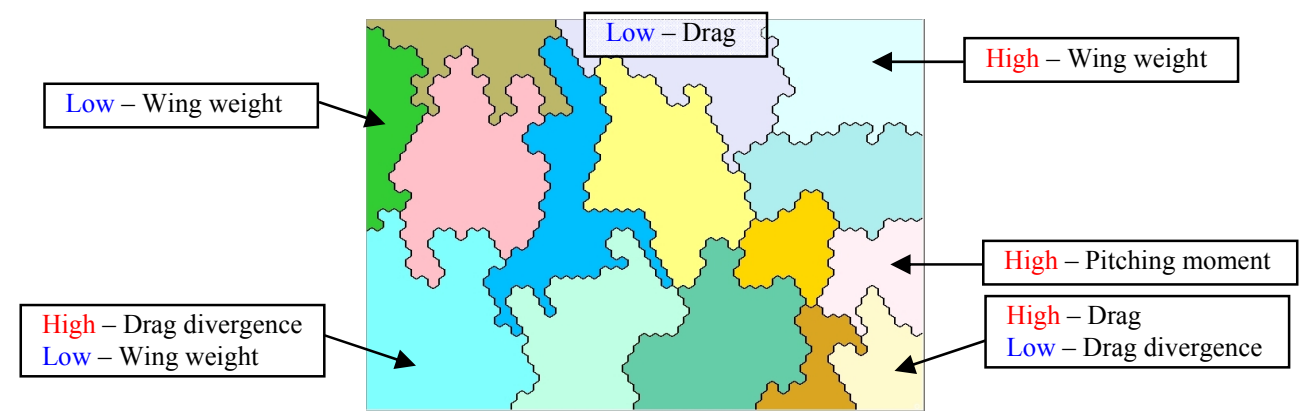

Figure 13. SOM of non-dominated solutions in the four-dimensional objective function space.

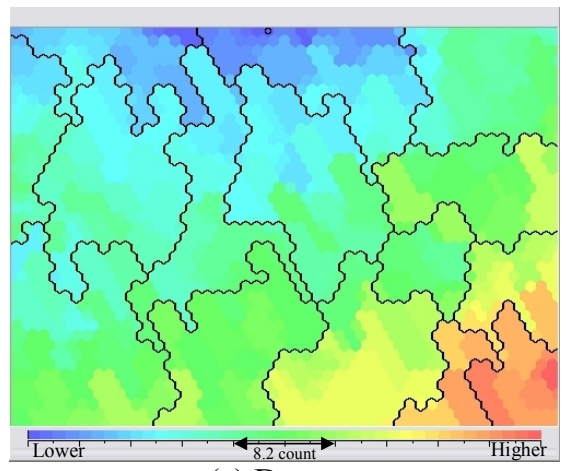

(a) Drag

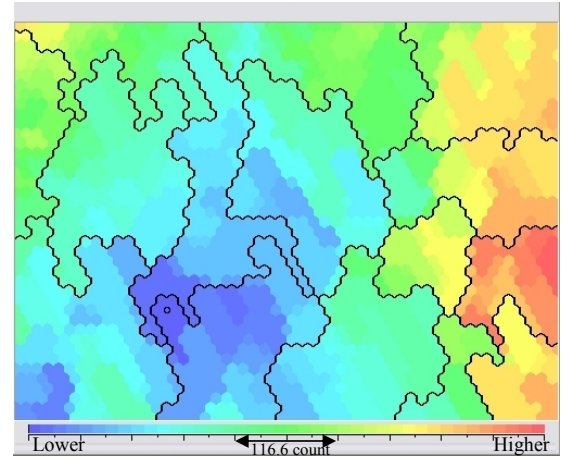

(c) Pitching moment

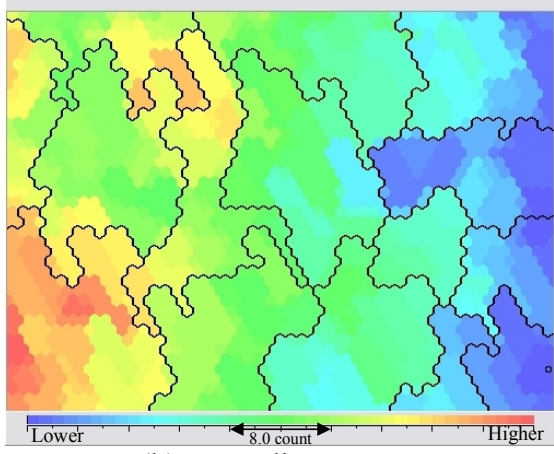

(b) Drag divergence

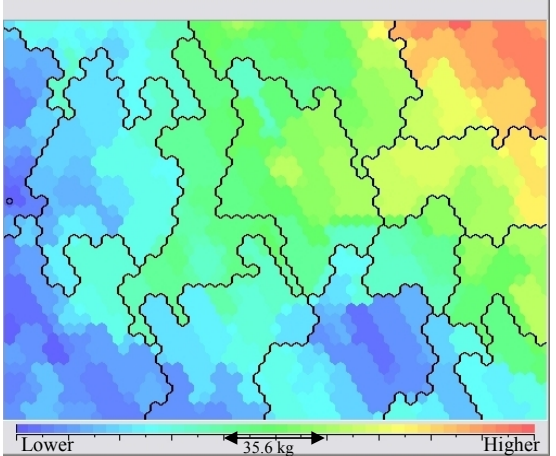

(d) Wing weight

Figure 14. SOM of non-dominated solutions colored by the objective functions. 
Next, solutions uniformly distributed in the design space have been projected onto the two-dimensional map of SOM. Figure 15 shows the resulting SOM with 13 clusters considering the four objectives. Furthermore, Fig. 16 shows the same SOM colored by the four objectives, respectively. These color figures show that the SOM indicated in Fig. 15 can be grouped as follows:

The right edge area corresponds to the designs with low drag divergence and low wing weight. The upper right area corresponds to those with high drag divergence. The upper left corner corresponds to those with high drag and high pitching moment. The lower left corner corresponds to those with low drag divergence and high wing weight.

As a result, there is no sweet spot in this design space that improves all four design objectives. If the drag divergence is tolerable, the right edge area will be a sweet spot for design.

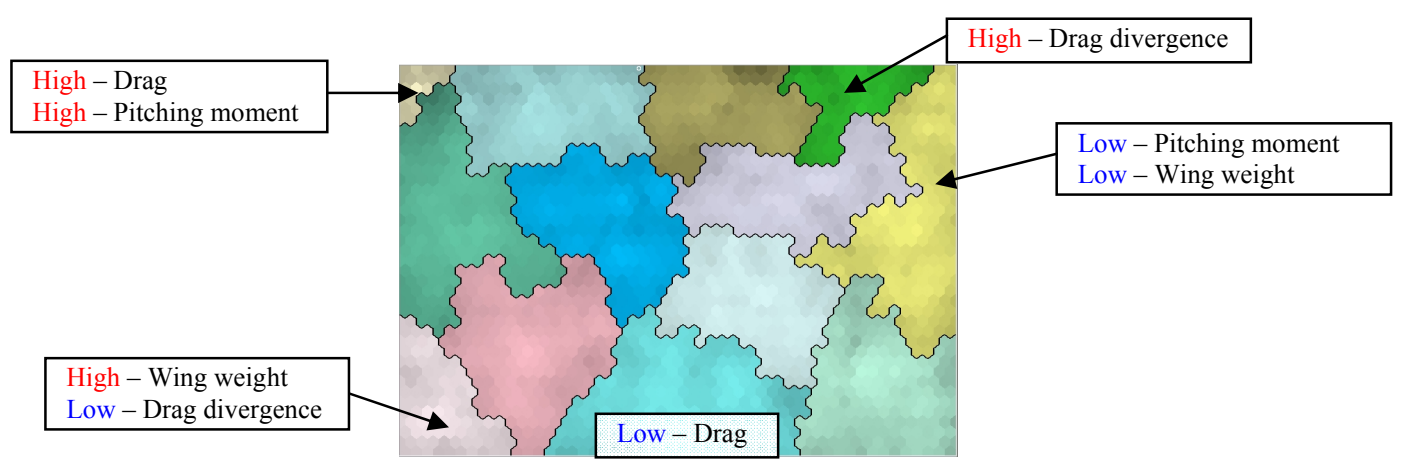

Figure 15. SOM of solutions uniformly distributed in the design space.

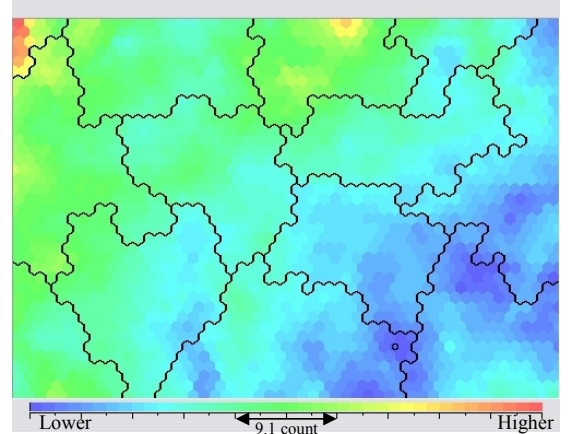

(a) Drag

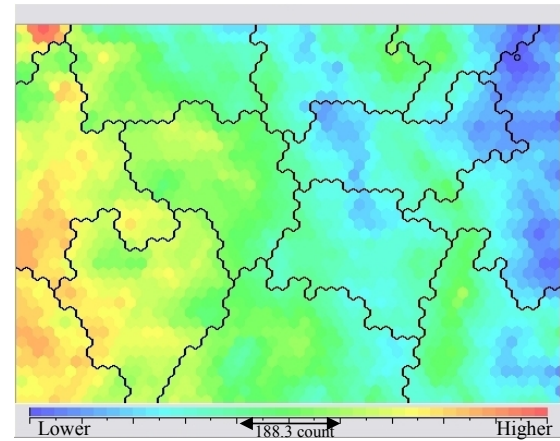

(c) Pitching moment

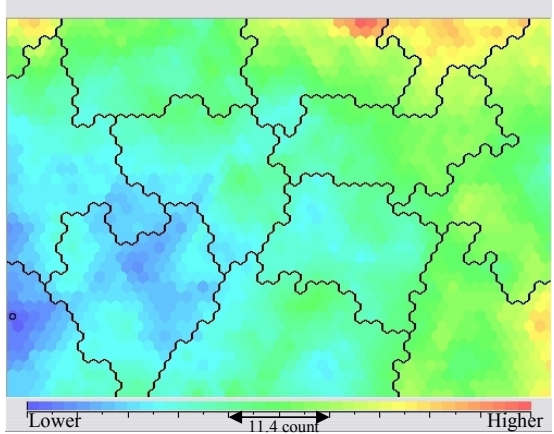

(b) Drag divergence

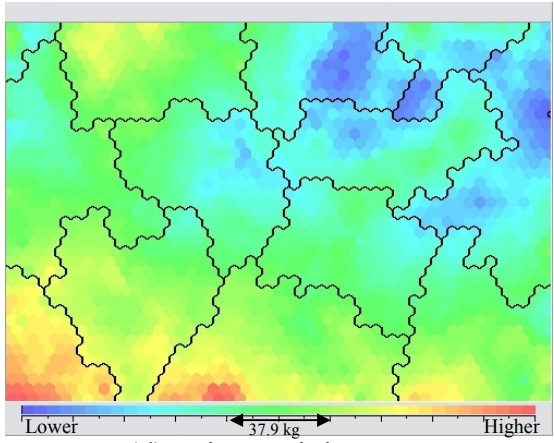

(d) Wing weight

Figure 16. SOM of solutions uniformly distributed in the design space colored by the objective functions. 


\section{Conclusion}

In order to construct the practical MDO design system for aircraft, following techniques are adopted in this study:

1) NURBS airfoil shape expression method

2) Optimizer based on Kriging model + MOGA

3) Robust computational mesh generation system

Furthermore, in order to evaluate aerodynamic and structural performance, CFD (full potential/Euler) and CSD (NASTRAN) coupling module was applied.

The present system was applied to the wing design problem that considers the aerodynamic and structural performance simultaneously. As a result of optimization, non-dominated solutions were generated with 160 function evaluations. One of the non-dominated solutions is found to be reduced by 6.2 counts in drag, 0.4 counts in drag divergence, 79.4 counts in pitching moment, and $74.0 \mathrm{~kg}$ in wing weight compared with the baseline shape. Therefore, it has been verified that the revised MDO system is very useful.

Moreover, Data Mining for the design space was performed using SOMs. In the case of SOM obtained from non-dominated solutions, it was found that there were trade-off relations between the drag divergence and the wing weight and between the drag divergence and the pitching moment. In the case of SOM obtained from solutions uniformly distributed in the design space, it was found that sweet spot exist if the drag divergence is tolerable. Data Mining using SOM provides knowledge regarding the design space and may salvage lost information during the optimization operation.

\section{Acknowledgments}

The Euler computations were performed using NEC SX-8 in the Institute of Fluid Science, Tohoku University. The authors would like to acknowledge Advanced Fluid Information Research Center, Institute of Fluid Science, for providing the computer resources.

\section{References}

${ }^{1}$ Kroo, I., Altus, S., Braun, R., Gage, P., and Sobieski, I., "Multidisciplinary Optimization Methods for Aircraft Preliminary Design,” AIAA Paper 94-4325-CP, 1994.

${ }^{2}$ Sobieszczanski-Sobieski, J. and Haftka, R. T., "Multidisciplinary Aerospace Design Optimization: Survey of Recent Developments," Structural Optimization, Vol.14, No.1, 1997, pp. 1-23.

3“NEDO" website, URL:http://www.nedo.go.jp/.

4"MSC Software" website, URL: http://www.mscsoftware.com/.

${ }^{5}$ Chiba, K., Obayashi, S., Nakahashi, K. and Morino, H., "High-Fidelity Multidisciplinary Design Optimization of Aerostructural Wing Shape for Regional Jet," AIAA paper 2005-5080, June 2005.

${ }^{6}$ Oyama, A., Obayashi, S., Nakahashi, K., and Hirose, N., "Aerodynamic Wing Optimization via Evolutionary Algorithms Based on Structured Coding," Computational Fluid Dynamics Journal, Vol.8, No.4, 2000, pp. 570-577.

${ }_{7}^{7}$ Lepine, J., Guibault, F., Trepanier, J-Y., and Pepin, "Optimized Nonuniform Rational B-spline Geometrical Representation for Aerodynamic Design of Wings,” AIAA Journal, Vol. 39, 2001, pp. 2033-2041.

${ }^{8}$ Takenaka, K., Obayashi, S., Nakahashi, K., Matsushima, K., "The Application of MDO Technologies to the Design of a High Performance Small Jet Aircraft - Lessons learned and some practical concerns -," AIAA paper 2005-4797, June 2005.

${ }^{9}$ Donald, R. J., Matthias S. and William J. W., "Efficient Global Optimization of Expensive Black-Box Function," Journal of global optimization, Vol.13, 1998, pp. 455-492.

${ }^{10}$ Jeong, S., Murayama, M., and Yamamoto, K., "Efficient Optimization Design Method Using Kriging Model," Journal of Aircraft, Vol.42, 2005, pp. 413-420.

${ }^{11}$ Matthias, S., "Computer Experiments and Global Optimization," Ph.D Dissertation, Statistic and Actuarial Science Dept., University of Waterloo, Waterloo, Ontario, 1997.

${ }^{12}$ Mckay, M. D., Beckman, R. J., and Conover, W. J., "A Comparison of Three Methods for Selecting Values of Input Variables in the Analysis of Output from a Computer Code," Technometric, Vol. 21, No. 2, 1979, pp. 239-245.

${ }^{13}$ Murayama, M., Nakahashi, K., and Matsushima, K., "Unstructured Dynamic Mesh for Large Movement and Deformation," AIAA Paper 2002-0122, 2002. 
${ }^{14}$ Yamazaki, W., Matsushima, K., and Nakahashi, K., "Aerodynamic Optimization of NEXST-1 SST Model at Near-Sonic Regime," AIAA Paper 2004-0034, 2004.

${ }^{15}$ Ito, Y. and Nakahashi, K., "Direct Surface Triangulation Using Stereolithography Data," AIAA Journal, Vol. 40, No. 3, 2002, pp. 490-496.

${ }^{16}$ Sharov, D. and Nakahashi, K., “A Boundary Recovery Algorithm for Delaunay Tetrahedral Meshing,” Proceedings of the 5th International Conference on Numerical Grid Generation in Computational Field Simulations, 1996, pp. $229-238$.

${ }^{17}$ Kohonen, T., Self-Organizing Maps, Springer, Berlin, Heidelberg, 1995.

${ }^{18}$ Jeong, S., Chiba, K., and Obayashi, S., "Data Mining for Aerodynamic Design Space," Journal of Aerospace Computing, Information, and Communication, Vol. 2, 2005, pp. 452-469. 